\title{
THE EFFECT OF PLASMA SHAPE ON H-MODE PEDESTAL CHARACTERISTICS ON DIII-D
}

\author{
by \\ T.H. OSBORNE, J.R. FERRON, R.J. GROEBNER, L.L. LAO, \\ A.W. LEONARD, R. MAINGI,1 R.L. MILLER, A.D. TURNBULL \\ M.R. WADE, 1 and J.G. WATKINS2
}

This is a preprint of a paper presented at the 4th IAEA Technical Committee Meeting on $\mathrm{H}$-mode and Transport Barrier, September 27-29, 1999, Oxford, United Kingdom, and to be printed in Plasma Physics and Controlled Fusion.

Work supported by U.S. Department of Energy under Contracts DE-AC03-99ER54463, DE-AC05-960R22464, and DE-AC04-94AL85000

10ak Ridge National Laboratory

2Sandia National Laboratories

\section{GENERAL ATOMICS PROJECT 30033} DECEMBER 1999 


\section{DISCLAIMER}

This report was prepared as an account of work sponsored by an agency of the United States Government. Neither the United States Government nor any agency thereof, nor any of their employees, make any warranty, express or implied, or assumes any legal liability or responsibility for the accuracy, completeness, or usefulness of any information, apparatus, product, or process disclosed, or represents that its use would not infringe privately owned rights. Reference herein to any specific commercial product, process, or service by trade name, trademark, manufacturer, or otherwise does not necessarily constitute or imply its endorsement, recommendation, or favoring by the United States Government or any agency thereof. The views and opinions of authors expressed herein do not necessarily state or reflect those of the United States Government or any agency thereof. 


\section{DISCLAIMER}

Portions of this document may be illegible in electronic image products. Images are produced from the best available original document. 


\title{
The Effect of Plasma Shape on H-mode Pedestal Characteristics on DIII-D
}

\author{
T.H. Osborne, J.R. Ferron, R.J. Groebner, L.L. Lao, A.W. Leonard, \\ M.A. Mahdavi, R. Maingi, ${ }^{1}$ R.L. Miller, ${ }^{2}$ A.D. Turnbull, M.R. Wade, ${ }^{1}$ J.G. Watkins ${ }^{3}$ \\ General Atomics, P.O. Box 85608, San Diego, CA 92186-5608 USA
}

\begin{abstract}
The characteristics of the H-mode are studied in discharges with varying triangularity and squareness. The pressure at the top of the H-mode pedestal increases strongly with triangularity primarily due to an increase in the margin by which the edge pressure gradient exceeds the ideal ballooning mode first stability limit. Two models are considered for how the edge may exceed the ballooning mode limit. In one model [1], access to the ballooning mode second stable regime allows the edge pressure gradient and associated bootstrap current to continue to increase until an edge localized, low toroidal mode number, ideal kink mode is destabilized. In the second model [2], the finite width of the $\mathrm{H}$-mode transport barrier, and diamagnetic effects raise the pressure gradient limit above the ballooning mode limit. We observe a weak inverse dependence of the width of the $\mathrm{H}$-mode transport barrier, $\Delta$, on triangularity relative to the previously obtained [3] scaling $\Delta \propto\left(\beta_{\mathrm{P}}^{\mathrm{PED}}\right)^{1 / 2}$. The energy loss for Type I ELMs increases with triangularity in proportion to the pedestal energy increase. The temperature profile is found to respond stiffly to changes in $\mathrm{T}^{\mathrm{PED}}$ at low temperature, while at high temperature the response is additive. The response of the density profile is also found to play a role in the response of the total stored energy to changes in the $\mathrm{W}^{\mathrm{PED}}$.
\end{abstract}

Note: As the first author submitted both his original manuscript and the text and figure changes directly to conference personnel without any publications processing at General Atomics, this version of his paper is not the same as that to be published in Plasma Physics and Controlled Fusion.

\footnotetext{
1Oak Ridge National Laboratory, Oak Ridge, Tennessee.

2Present address: Archimedes Technology Group, San Diego, California.

${ }^{3}$ Sandia National Laboratories, Albuquerque, New Mexico.
} 


\section{Introduction}

Although the $\mathrm{H}$-mode transport barrier is localized to the plasma edge, this region plays a significant role in overall plasma performance and in heat transport to the divertor through ELMs. The variation of the $\mathrm{H}$-mode pedestal with shape is therefore an important consideration in the design of future tokamaks.

In discharges without internal transport barriers, the pedestal temperature, $\mathrm{T}^{\mathrm{PED}}$, may influence the core plasma temperature profile through profile 'stiffness'. Stiff temperature profiles, in which the core temperature is proportional to the pedestal temperature, are expected from theory based transport models which include nonlinear, electrostatic ion temperature gradient (ITG) mode and trapped electron mode (TEM) physics $[4,5]$. In such models a high level of turbulence and transport is expected above a critical temperature gradient scale length. In other models [6] in which electron transport dominates a critical temperature gradient is expected rather than a gradient scale length, and therefore the core temperature should vary additively rather than proportionally with $\mathrm{T}^{\mathrm{PED}}$. Recent work suggests that the core profiles may change from an additive to stiff above a critical value of $\mathrm{T}^{\mathrm{PED}}$ [7]. The response of the density profile is also important in determining the effect of pedestal parameters on the total stored energy as will be discussed in Section 4.

The diffusivities of the H-mode transport barrier are typically so small that the $\mathrm{H}$-mode pedestal temperature and density are not set by transport, but rather by the edge localized mode, ELM [11], instability combined with the physics which sets the width of the H-mode transport barrier. Several classes of ELMs are identified. Type I, or giant, ELMs are the most prevalent at heating powers well above the $\mathrm{H}$-mode power threshold. Type I ELMs increase in frequency with increasing heating power and have lower toroidal mode number, $3<\mathrm{n}<10$, magnetic precursor modes which typically grow on an ideal time scale. The energy loss from Type I ELMs appears in the divertor on a millisecond time scale, and scaling from present tokamaks[8] indicates that they will be very large, 10-100 MJ/ELM in a machine like ITER[8], and therefore of great concern for divertor design. We will mainly discuss discharges in the Type I ELM regime in this paper. Type III ELMs are observed both in a low density $[9,10]$ and low temperature regimes [11]. Precursor modes to Type III ELMs grow on a resistive MHD times scale and have similar mode number to 
those observed for Type I ELMs. Energy and particle confinements are generally lower with Type III ELMs relative to Type I ELM discharges.

This paper primarily describes the effect on the H-mode pedestal of the variation of the upper triangularity in lower single null, divertor discharges on DIII-D. In Section 2 of this paper we discuss the effect of plasma shape on the edge pressure gradient. In Section 3 we discuss the effect of shape on the H-mode barrier width. In Section 4, we examine the behavior of the pedestal at high density for different shapes, look at the effect of the pedestal parameters on the core profiles, and discuss the effect of shaping on ELM energy loss. Section 5 contains a summary and discussion of the results. 


\section{The Effect of Plasma Shape on Edge Pressure Gradient}

In single null divertor discharges with Type I ELMs the pressure at the top of the $\mathrm{H}$-mode pedestal, $\mathrm{p}^{\mathrm{PED}}$, is observed to increase by nearly a factor of 2 in going from upper triangularity, $\delta_{\text {UPPER }}$, of 0.0 to 0.5 [Fig. 1(a)]. The increase in pressure is primarily due to an increase in edge pressure gradient [Fig. 1(b)], while the width of the H-mode transport barrier, $\Delta_{\mathrm{pe}}$, remains relatively constant [Fig. 1(c)]. Profiles were measured with Thomson scattering for electrons [12] and charge exchange spectroscopy for ions [13]. The Thomson scattering system uses eight YAG lasers each firing at $20 \mathrm{~Hz}$. For these experiments, four lasers were devoted to the vertical chord which measures from $\mathrm{r} / \mathrm{a}=0.3$ to beyond the separatrix, with spatial resolution of roughly $0.6 \mathrm{~cm}$ near the separatrix when mapped to outer midplane. Three of the remaining lasers were devoted to central measurements along a horizontal chord and the remaining laser was available for divertor measurements, so that high-resolution edge measurements were made every $12 \mathrm{~ms}$. The CER system is normally tuned to $\mathrm{C}^{6+}$ light since carbon is the main impurity ion in DIII-D. The system is absolutely calibrated and is used to obtain edge $T_{I}$ and $Z_{\text {EFF }}$ with spatial resolution of $0.4 \mathrm{~cm}$ on the outboard midplane. The CER system was run with 5 to $10 \mathrm{~ms}$ time resolution that averages over ELMs in some cases. Edge profile characteristics were determined from these measurements by fitting the data to a hyperbolic tangent function [14]. In general the width of the steep gradient region in the ion pressure is found to be equal to that of the electron pressure, and the more easily obtained electron pressure gradient scale length is used in most cases.

A comparison of the measured pressure gradient with the stability limit for ideal, infinite $\mathbf{n}$ ballooning modes was made using the BALOO [15] stability code. These calculations show that the ballooning mode stability limit is insensitive to the triangularity change, so that the increase in the maximum pressure gradient in the H-mode pedestal, $\nabla \mathrm{p}^{\mathrm{PED}}$, with $\delta_{\text {UPPER }}$ represents an increase in the margin by which $\nabla \mathrm{p}^{\mathrm{PED}}$ exceeds the ballooning mode critical pressure gradient [Fig. 1(b)]. Even at low triangularity the measured pressure gradient exceeds the ballooning mode limit by a large margin.

The stability of ballooning modes is usually described in terms of an S-_ diagram [16] where

$$
\mathrm{S}=2 \frac{\mathrm{V}}{\mathrm{q}} \frac{\partial \mathrm{q} / \partial \psi}{\partial \mathrm{V} / \partial \psi}
$$


is the magnetic shear, and

$$
\alpha=\frac{\mu_{0}}{2 \pi^{2}} \frac{\partial \mathrm{p}}{\partial \psi} \frac{\partial \mathrm{V}}{\partial \psi}\left(\frac{\mathrm{V}}{2 \pi^{2} \mathrm{R}}\right)^{1 / 2},
$$

is the normalized pressure gradient (which reduces to the familiar

$$
\alpha=\frac{\mathrm{Rq}^{2}}{\mathrm{~B}^{2} / 2 \mu_{0}} \frac{\partial \mathrm{p}}{\partial \mathrm{R}}
$$

in cylindrical geometry) where $\mathrm{q}$ is the safety factor, $\mathrm{V}$ the plasma volume, $\psi$ the poloidal flux, and $\mathrm{R}$ the major radius and $\mathrm{B}$ the toroidal field. In the first stable regime the marginal stability curve is given by $\alpha_{\mathrm{CRIT}}^{\mathrm{BAL}}=\mathrm{f}(\mathrm{S})$, where $\mathrm{f}$ is an increasing function of $\mathrm{S}$, and the plasma is unstable at higher-pressure gradients. For the strongly shaped discharges studied is a very weak function of $\mathrm{S}$ near the boundary and is roughly constant for a given shape. Below a critical value of $S$ in noncircular cross-section tokamaks, which scales as $\mathrm{S}_{\mathrm{sS}} / \mathrm{q}^{2} \equiv \mathrm{C}_{\mathrm{SS}}$ where $\mathrm{C}_{\mathrm{SS}}$ varies with shape, a region with no pressure gradient limit, referred to as the second stable regime, is encountered. For $\delta=0.25, \mathrm{C}_{\mathrm{SS}} \approx 0.2$. S is reduced when the edge current, $\mathrm{j}_{\mathrm{TOR}}^{\mathrm{PED}}$, increases as

$$
\mathrm{S} \approx \mathrm{S}_{0}-\frac{\mu_{0} \mathrm{a}}{\mathrm{B}_{\mathrm{P}}} \mathrm{j}_{\mathrm{TOR}}^{\mathrm{PED}} \text {. }
$$

For the discharge shapes studied, $S_{0} \approx 6$ is the value of $S$ with no edge current. Even with $\mathrm{j}_{\mathrm{TOR}}^{\mathrm{PED}}=0$, discharges with $\mathrm{q}_{95}>5.5$ would be expected to have no ballooning mode pressure gradient limit near the edge. $C_{S S}$ roughly doubles in going from $\delta$ of 0.0 to 0.5 , so that higher triangularity discharges would be expected to have no pressure gradient limit to lower $q$ values; and $\mathrm{C}_{\mathrm{ss}}$ is also reduced with increasing edge current. For $\mathrm{q}^{2}<\mathrm{S}_{0} / \mathrm{C}_{\mathrm{sS}}$ access to the second stable regime is still possible for sufficiently large edge current density. For time scales long compared to an edge skin time $(\approx 50 \mathrm{~ms})$, the bootstrap current associated with the high edge pressure gradient is expected to be much larger than the Ohmic contribution, in this case $S$ is given by

$$
\mathrm{S} \approx \mathrm{S}_{0}-\frac{\mathrm{C}_{\mathrm{BS}}}{\varepsilon^{1 / 2}} \alpha
$$

Here $\mathrm{C}_{\mathrm{BS}}$ contains the effect of cross-sectional shape on trapped particle fraction (not a strong effect) and is reduced by collisions

$$
\mathrm{C}_{\mathrm{BS}} \approx \frac{1}{1+v_{*}^{1 / 2}}
$$

For second stable access afforded by edge current,

$$
\mathrm{S}_{\mathrm{SS}}>\mathrm{S}_{0}-\frac{\mathrm{C}_{\mathrm{BS}}}{\varepsilon^{1 / 2}} \alpha_{\mathrm{CRIT}}
$$


or

$$
\mathrm{q}>\mathrm{q}_{\mathrm{SS}}^{\mathrm{MIN}} \equiv\left(\frac{\mathrm{S}_{0}-\mathrm{C}_{\mathrm{BS}} \alpha_{\mathrm{CRIT}} \varepsilon^{-1 / 2}}{\mathrm{C}_{\mathrm{SS}}}\right)^{1 / 2}
$$

For typical values of DIII-D, with $\mathrm{C}_{\mathrm{BS}}=1$, we can estimate $\mathrm{q}_{\mathrm{SS}}^{\mathrm{MIN}} \approx 3.5$. More detailed estimates by Miller [1] indicate that $\mathrm{q}_{\mathrm{SS}}^{\mathrm{MIN}} \approx 2.5$ for $\kappa=1.8$ and $\delta=0.4$, and $\mathrm{q}_{\mathrm{SS}}^{\mathrm{MN}} \approx 3.5$ for at $\delta=0.0$. These estimates indicate that most of the moderate $q$ discharges with moderate shaping on DIII-D should have access to second stability in the H-mode pedestal region.

Whether or not a lower $\mathrm{q}$ discharge has access to second stability depends on accurately determining $\mathrm{j}_{\mathrm{TOR}}^{\mathrm{PED}}$. The current density profile in DIII-D is determined in fitting the MHD equilibrium to data from magnetic probes, the motional Stark effect diagnostic [17], and kinetic profile diagnostics. The MSE signal is dominated by the contribution from the large radial electric field in the H-mode edge region. As a result, is not possible at present to measure the edge current density directly with sufficient accuracy to determine if second stable access is open for low q discharges. By constraining the edge current with values obtained from transport simulations using the ONETWO code, we find that discharges with $\delta_{\text {UPPER }}>0.2$ have second regime access down to $q_{95} \approx 3$. For $\delta_{\text {UPPER }}=0.0$, whether or not second stable access is predicted to be possible depends on the bootstrap current model and the extent to which the ohmic current contribution is relaxed in the edge region.

In support of the view that edge second stability access allows $\alpha>\alpha_{\mathrm{CRIT}}^{\mathrm{BAL}}$ are experiments in which the plasma 'squareness' was varied [18]. In these experiments a sudden transition to small high frequency ELMs was observed at very high and very low squareness where simulations [1] predicted edge current density significantly exceeding the collisionless bootstrap current would be required for access to second stability [Fig. 2(a)]. This qualitative change in ELM character occurred over a narrow range in squareness and as such is unlikely to be the result of a change in fueling efficiency or impurity influx. At the transition to small ELMs the edge pressure gradient is observed to drop to the calculated first stable limit. Additional gas puffing in discharges which would otherwise have large ELMs but which are close to the small ELM shape produced a transition to small ELMs consistent with a decrease in edge bootstrap current with increased collisionality.

If access to the second stable regime for ballooning modes allows $\alpha>\alpha_{\mathrm{CRIT}}^{\mathrm{BAL}}$ another instability must be responsible for the ELM. The observation of modes with $3<\mathbf{n}<10$ 
growing on an ideal time scale as precursors to some Type I ELMs on DIII-D [19,30] and similar modes on Asdex-Upgrade [20] suggests low $\mathrm{n}$ ideal modes. Computations with the GATO [21] code indicate that the edge can be unstable to low $n$ surface localized kinks which become unstable above a critical value of the edge current density and increase in growth rate also with increasing pressure gradient. The lowest $\mathrm{n}$ modes are typically stable due to the effect of the conducting boundary and the mode's growth rate increases with $n$. At present the stability of $n \leq 6$ can be calculated in GATO and the behavior of higher $n$ modes is unclear. Recent simulations [22] indicate that the critical current density required for instability increases with increasing triangularity in qualitative agreement with the experimental results [Fig. 1(b)]. It is unclear at present why a ballooning mode like scaling which is observed for the pressure gradient before the Type I ELM on several machines $[3,23,24,25]$, would be expected for the low $n$ modes.

Recent work by Rogers [2] might also account for $\alpha>\alpha_{\mathrm{CRIT}}^{\mathrm{BAL}}$. Their nonlinear simulations are based on the on the Braginski equations for electrostatic and electromagnetic modes. Long wavelength modes, which would otherwise be destabilized by the high pressure gradient, are stabilized when the extent of the steep gradient region is small compared to the radial extent of the mode. Short wavelength modes are stabilized by diamagnetic effects. This results in a critical pressure gradient that increases as the extent of the H-mode transport barrier diminishes as

$$
\alpha_{\mathrm{CRIT}} \approx \alpha_{\mathrm{CRIT}}^{\mathrm{BALL}} \cdot \frac{\rho_{\mathrm{i}}}{\Delta}\left(\frac{2}{1+1 / \tau} \frac{\mathrm{R}}{\Delta}\right)^{1 / 2},
$$

where is the ion gyro radius, $\tau=T_{i} / T_{e}$, and $\Delta$ is the width of the steep gradient region. This result is consistent with the envelope of the DIII-D data for a fixed shape [Fig. 2(b)], however it does not account for the variation of $\alpha$ with triangularity or the low pressure gradient observed in the high and low squareness discharges. 


\section{The Effect of Plasma Shape on H-mode Transport Barrier Width}

The $\mathrm{H}$-mode transport barrier is thought to be the result of the suppression of turbulence in the plasma edge. A region of sheared $E_{R} \times B$ flow in the edge is observed experimentally with sufficient magnitude to suppress observed fluctuations [26]. The width of the H-mode transport barrier may be set by the innermost point at which turbulence suppression occurs. In the results presented here the H-mode transport barrier is assumed to coincide with the steep electron pressure gradient region, and the scaling relations presented here apply to the interval between Type I ELMs. We have previously [3] tested models for the barrier width which are determined by: (1) the ion orbit loss [27] which results in $\Delta \propto \rho_{\mathrm{i}}^{\mathrm{POL}}$, where $\rho_{\mathrm{i}}^{\mathrm{POL}}$ is the poloidal gyro radius, and (2) a balance between velocity shearing rate and ITG mode growth rate that results in $\Delta \propto \rho_{\mathrm{i}}$, with experiments that vary $\mathrm{T}^{\mathrm{PED}}$ by gas puffing and divertor pumping. These experiments indicated no direct dependence of $\Delta_{\mathrm{pe}}$ on temperature. Fitting a database of discharges with fixed shape we arrived at the scaling $\Delta_{\mathrm{pe}} \propto\left(\beta_{\mathrm{P}}^{\mathrm{PED}}\right)^{1 / 2}$. This scaling can also roughly be written as $\Delta_{\mathrm{pe}} \propto \alpha$ which is suggestive of the model of Drake and Rogers [28] in which a critical $\alpha$ is required for the $\mathrm{L} \rightarrow \mathrm{H}$ transition in a regime where resistive modes are important. Comparing this scaling with discharges at different triangularities we find that the measured $\Delta_{\mathrm{pe}}$ is below the scaling predictions at high trangularity and above the scaling at low triangularity indicating an inverse triangularity dependence (Fig. 3). 


\section{Effect of Shape on Overall Plasma Performance}

The behavior of the $\mathrm{H}$-mode pedestal and overall plasma performance was studied in $\mathrm{D}_{2}$ gas puff fueled discharges at different triangularities. With no additional gas puff during $\mathrm{H}$-mode, we find that the energy loss from Type I ELMs, $\triangle \mathrm{E}_{\mathrm{ELM}}$, increases with triangularity in proportion to the pedestal energy increase. $\Delta \mathrm{E}_{\mathrm{ELM}}$ can be greatly reduced with gas puffing however without, in some cases, any significant reduction in energy confinement.

With $\mathrm{D}_{2}$ gas puffing during the $\mathrm{H}$-mode phase, we find that the pedestal pressure begins to decrease above a density of $n_{e}^{P E D} / n_{G}=0.8$, where $n_{G}=I_{P} / \pi a^{2}$ is the Greenwald density, in both low and high triangularity discharges (Fig 4.). This reduction in pedestal pressure at high density is due to the reduction in the edge pressure gradient with temperature, beginning at a higher temperature at higher triangularity, but the decay of the pressure is at roughly the same rate, $p \propto \eta^{-0.5}$, where $\eta$ is the resistivity, at both triangularities. The transport barrier width remains relatively constant with temperature as discussed in the previous section. This rate of reduction is closer to what might be expected for tearing modes, where $\gamma \propto \eta^{3 / 5}$, than for resistive ballooning modes, where $\gamma \propto \eta^{1 / 3}$.

The pedestal pressure with Type III ELMs at both low and high density is limited to less than $1 / 2$ of the Type I value [Fig. 4(b)]. We have previously shown [9] that in the low-density regime, Type III ELMs do not exist above a critical value of $\alpha$. This appears to be the case for the high-density branch of Type III ELMs on DIII-D. At the transition to Type III ELMs brought about by excessive gas puffing at too low a heating power a sudden reduction in edge pressure gradient is observed.

The change in global energy confinement in response to changes in the pedestal pressure involves several factors. The response of the core temperature profile to changes in the pedestal temperature varies with temperature [Fig. 5(a)]. At low temperature the profile behaves rigidly with $T(r) \propto T^{P E D} \cdot f(r)$, while at high temperature the profile is additive, $T(r) \propto T^{\mathrm{PED}}+f(r)$. This result is in qualitative agreement with a recent model by Janaschitz [7] in which at low temperature transport is dominated by ITG modes with a critical temperature gradient scale length, while at high temperature Rebut-Lallia electron transport characterized by a critical gradient dominates. Low and high triangularity discharges at low density with the same heating power and plasma current were found to differ in stored energy by the difference in pedestal energy, $W^{\mathrm{PED}}=\mathrm{p}^{\mathrm{PED}} \mathrm{V}$ as expected for 
an additive temperature profile and similarly flat density profiles. For densities near the Greenwald density on DIII-D all discharges studied were in the stiff profile, low temperature regime. In this regime for fixed density profile $\mathrm{W}^{\mathrm{TOTAL}} \propto \mathrm{W}^{\mathrm{PED}}$ so that the energy confinement is sensitive to a reduction in the pedestal pressure. However the evolution of the density profile also plays a role in the response of the core plasma to changes in pedestal parameters in the stiff profile regime. In the extreme case that all the added density appears only in the pedestal then, for a constant $\mathrm{p}^{\mathrm{PED}}, \mathrm{W}^{\mathrm{TOTAL}} \propto \mathrm{T}^{\mathrm{PED}}$ and a very large reduction in stored energy is expected, while if the density profile becomes more peaked at high density the total stored energy can increase with density. The importance of the response of the density profile can be seen dramatically in some gas puff fueled discharges wit spontaneous density peaking [Fig. 5(b-e)] where stored energy and $\mathrm{H}$ factor increase with density. In these discharges, the increase in $\mathrm{W}^{\text {TOTAL }}$ cannot be accounted for by assuming $\mathrm{W}^{\text {TOTAL }} \propto \mathrm{T}^{\mathrm{PED}}$ (stiff temperature and flat density profiles) or $\mathrm{W}^{\mathrm{TOTAL}}=\mathrm{W}^{\mathrm{L}-\mathrm{MODE}}+\mathrm{W}^{\mathrm{PED}}$ (L-mode core plus the pedestal energy). 


\section{Summary and Discussion}

In the majority of discharges on DIII-D the pressure gradient before a Type I ELM exceeds the ideal ballooning mode first stability limit by a large factor which increases with increasing triangularity. A possible explanation for this result is access to the second stable regime, which results from the plasma shaping and large edge bootstrap current. The ELM may then be triggered by lower $\mathrm{n}$ surface kinks that are destabilized by both current density and pressure gradient. This model is most strongly supported by the transition to small ELMs and drop of the pressure gradient to the first stable limit at high and low squareness. Quantitative comparison between theory and experiment however will not be possible until improved edge current density measurements are available. Although the effect of the finite width of the H-mode transport barrier on stability [2] is not at present consistent with the squareness experiment, this theory is only currently developed for circular cross-section. In the highly non-circular DIII-D cross-section second stability effects may also appear in this model. Moreover, it is expected that finite $\Delta$ will improve the low $\mathrm{n}$, ideal edge kink stability and this is currently under investigation in low n kink stability studies.

The width of the H-mode transport barrier is not increased with triangularity as much as is predicted from a scaling derived from a fixed shape database. Large differences in the experimental results in this area are obtained for the various machines. On JT-60U $\Delta \propto \rho_{i}^{\mathrm{POL}}$, and $\Delta \propto \rho_{\mathrm{i}}$ for JET is inferred from the variation of pedestal pressure, while a temperature scaling seems to be ruled out in the DIII-D results. Also, $\Delta$ is roughly a factor of two larger on Asdex-Upgrade compared to DIII-D, even though the machines are very similar in size and shaping. Since the inner edge of the H-mode transport barrier represents a transition point it is possible that differences in the instabilities driving the turbulence or in the mechanism for the radial electric field generation might account for the different scalings obtained in different devices. For example if ITG modes dominate at the inner edge of the JET barrier $\Delta \propto \rho_{\mathrm{i}}$ might be expected, on JT-60U perhaps the large ripple losses drive the electric field resulting in $\Delta \propto \rho_{i}^{\text {POL }}$, while at the lower temperatures on DIII-D perhaps resistive modes are more important and $\Delta \propto \alpha$. Other models for the barrier width, such as a dependence on the particle source profile [29] should be tested experimentally.

The response of the core profiles to changes in the $\mathrm{H}$-mode pedestal involves

several factors. The temperature profile appears to react stiffly to changes in the $\mathrm{T}^{\mathrm{PED}}$ at low temperature while at high temperature the effect is only additive. At very high pedestal 
densities the pedestal pressure is reduced perhaps as a consequence of the low $\mathrm{n}$ ideal modes which may trigger the Type I ELM becoming resistive. If this is indeed the case, future devices with higher edge temperature may not suffer the degradation in energy confinement observed at high density in current experiments. The response of the density profile to changes in the pedestal is also an important factor. In some gas puff fueled discharges on DIII-D with spontaneous density peaking the $\mathrm{H}$ factor is higher with $\overline{\mathrm{n}}_{\mathrm{e}} / \mathrm{n}_{\mathrm{G}}>1$ than in discharges with no additional gas fueling. 


\section{Acknowledgment}

This is a report of work supported by the U.S. Department of Energy under Contract Nos. DE-AC03-99ER54463, DE-AC05-96OR22464, and DE-AC04-AL85000. 


\section{References}

[1] Miller R. L. et al. (1998) Plasma Phys. Contr. Fusion 40, 753.

[2] Rogers B. N. et al. (1999) Phys. Plasmas 6, 2797.

[3] OSBORNE T. H., et al. (1999) J. Nucl. Mater. 266-269, 131.

[4] Kotschenreuter M, et al. (1996) Proc. 16th Int. Conf. on Plasma Phys. and

Controlled Nucl. Fusion Research, Montreal, Canada, Vol. 2, p. 371.

[5] Waltz R. E., et al. (1998) Phys. Plasmas 5, 1784.

[6] REBUT P., et al. (1988) Proc. 12th Int. Conf. on Plasma Phys. and Controlled Nucl.

Fusion Research, Montreal, Canada, p.191.

[7] IGITKHANOV Yu, et al. (1999) H-Mode Workshop.

[8] LEONARD A. W., et al. (1997) J. Nucl. Mat. 241-243, 628.

[9] OSBORNE T. H., et al. (1998) Plasma Phys. Contr. Fusion 40, 845.

[10] SATORI R., et al. (1999) H-Mode Workshop.

[11] ZOHM H. (1996) Plasma Phys. Contr. Fusion 38, 105.

[12] CARLSTROM T. N. et al. (1992) Rev. Sci. Instrum. 63, 4901.

[13] GOHIL P., et al. (1992) Proc. 14th IEEE/NPSS Symposium on Fusion Engineering, California, 1991. Vol. 2, p.1199.

[14] Groebner R. J., et al. (1998) Phys. Plasmas 51800.

[15] MILLER R. L., et al. (1997) Phys. Plasmas 41062.

[16] GREENE J. W., et al. (1981) Nucl. Fusion 21, 453.

[17] RICE B. W., et al. (1997) Phys. Rev. Lett. 792694.

[18] FERRON J. R., et al. (1999) Proc. 26th EPS Conf. on Controlled Fusion and Plasma Physics, Maastricht, The Netherlands (to be published).

[19] OSBORNE T. H., et al. (1997) Proc. 24th EPS Conf. on Plasma Phys. and Contr. Fusion Research, Berchesgaden, Germany, Vol. 21A, Part III, p. 1101.

[20] ZoHM H., et al. (1996) Proc. 16th IAEA Conf. on Plasma Phys. and Contr. Nucl.

Fusion Research, Montreal, Canada, Vol. 1, p. 439.

[21] Bernard L. C. et al. (1981) Comp. Phys. Commun. 24, 377.

[22] SAARELMA S., et al. (1999) H-Mode Workshop.

[23] NAVE M. F. F., et al. (1999) H-Mode Workshop

[24] SUTTROP W., et al. (1996) Proc. 23th EPS Conf. on Plasma Phys. and Contr. Fusion Research, Kiev, Ukraine, Vol. 20C, Part I, p. 47.

[25] KaMAdA Y. (1996) Plasma Phys. Contr. Fusion 38, 1387. 
[26] BURRell K. H. (1997) Phys. Plasmas 41499.

[27] SHAING K. C. (1992) Phys. Fluids B 4290.

[28] RoGERS B. N., et al. (1997) Phys. Rev. Lett. 79229.

[29] STAebler G. M. (1998) Plasma Phys. Contr. Fusion 40, 569.

[30] STRAIT E.J., et al. (1993) Proc. 20th EPS Conf. on Plasma Phys. and Contr. Fusion Research, Lisbon, Portugal, Vol. 18B, Part I, p. 211. 


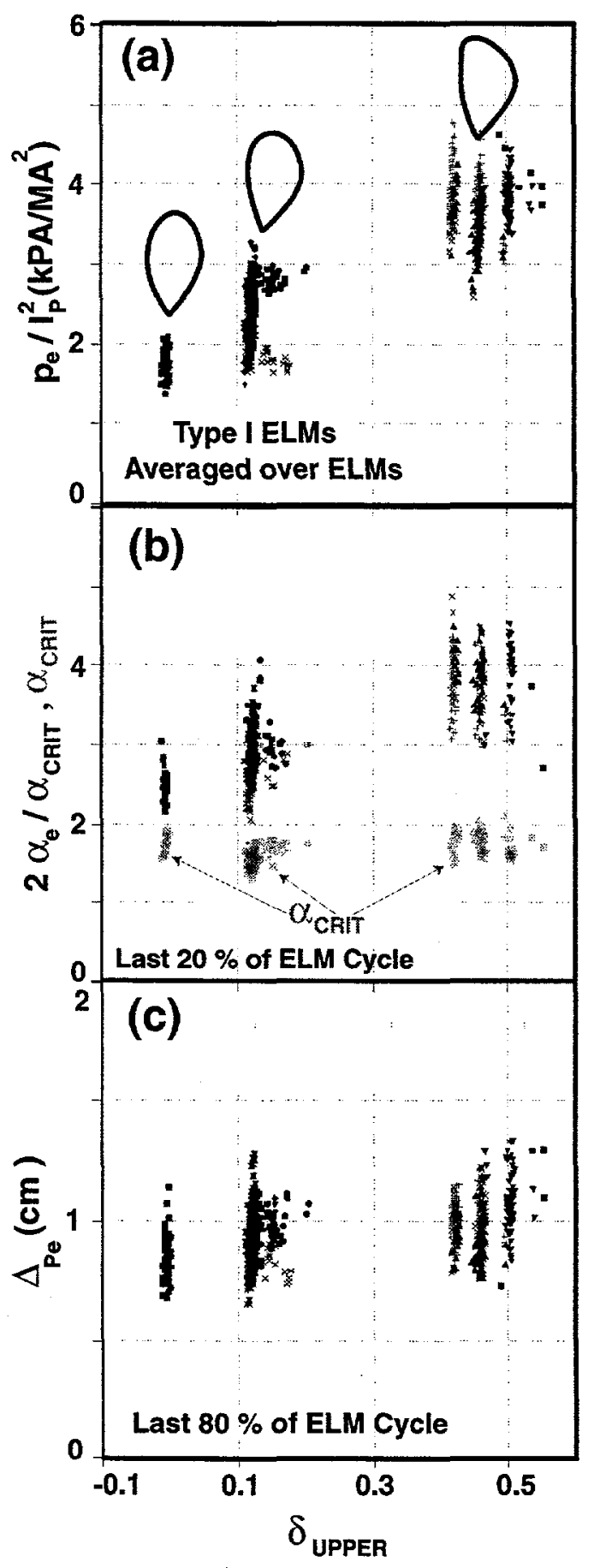

Fig. 1. Effect of triangularity, $\delta$, on the $\mathrm{H}$-mode pedestal. (a) Electron pressure at the top of the H-mode transport barrier showing a strong increase with $\delta$, (b) margin by which the edge pressure gradient exceeds the ballooning mode limit, $\alpha_{\mathrm{CRIT}}$, increases with $\delta$, (c) width of the electron pressure gradient edge steep gradient region, $\Delta_{\mathrm{pe}}$, is relatively independent of $\delta$. 


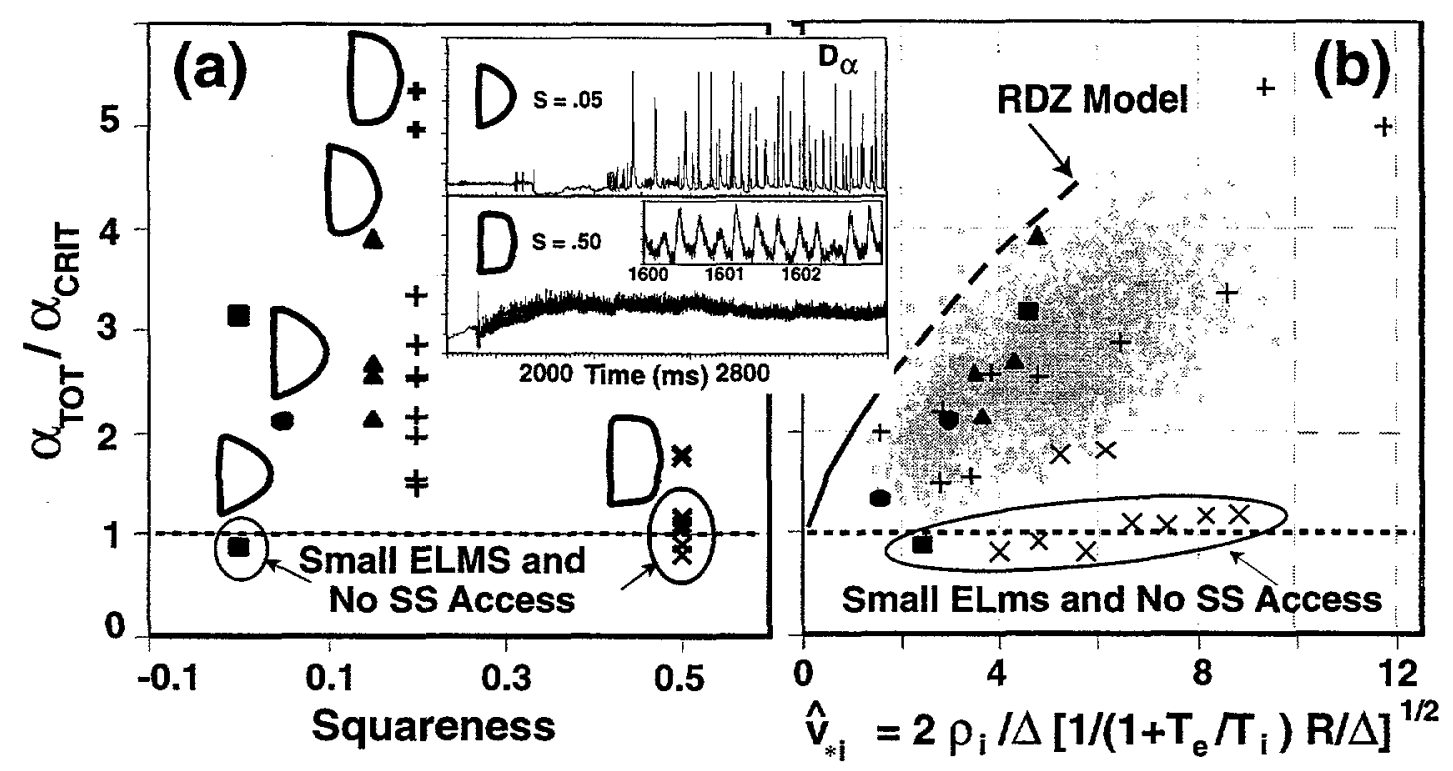

Fig. 2. Effect of squareness on edge pressure gradient relative to ballooning mode limit, $\alpha / \alpha_{\text {CRIT }}$ (a) At high and low squareness $\alpha / \alpha_{\mathrm{CRIT}}=1$ and small high frequency ELMs are observed where no access to the second stable regime is expected, (b) large ELM data is relatively consistent with Roger [2] model as was the original data base at for fixed shape (gray points), however small ELM discharges deviate from this scaling.

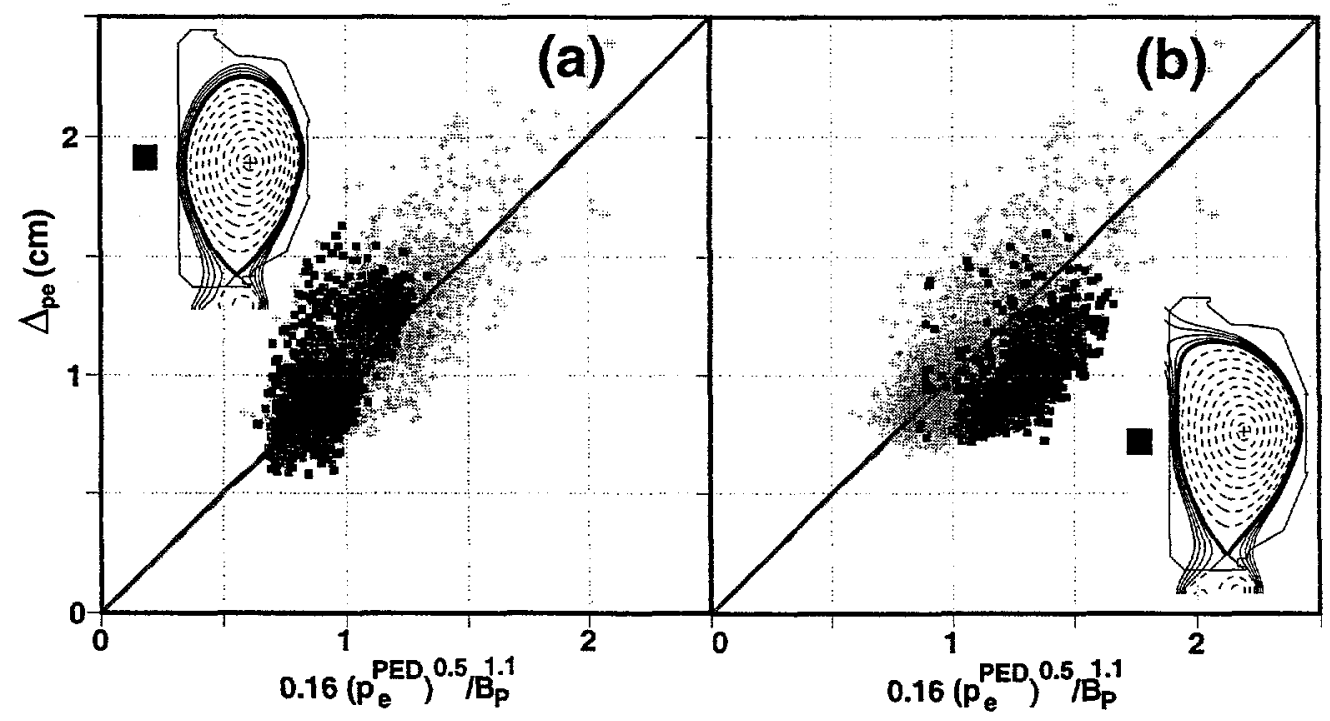

Fig. 3. Effect of triangularity on the H-mode transport barrier width, $\Delta_{\text {pe }}$, relative to scaling derived for fixed shape shows inverse dependence with triangularity. Gray points show original medium triangularity database from which scaling was derived. 


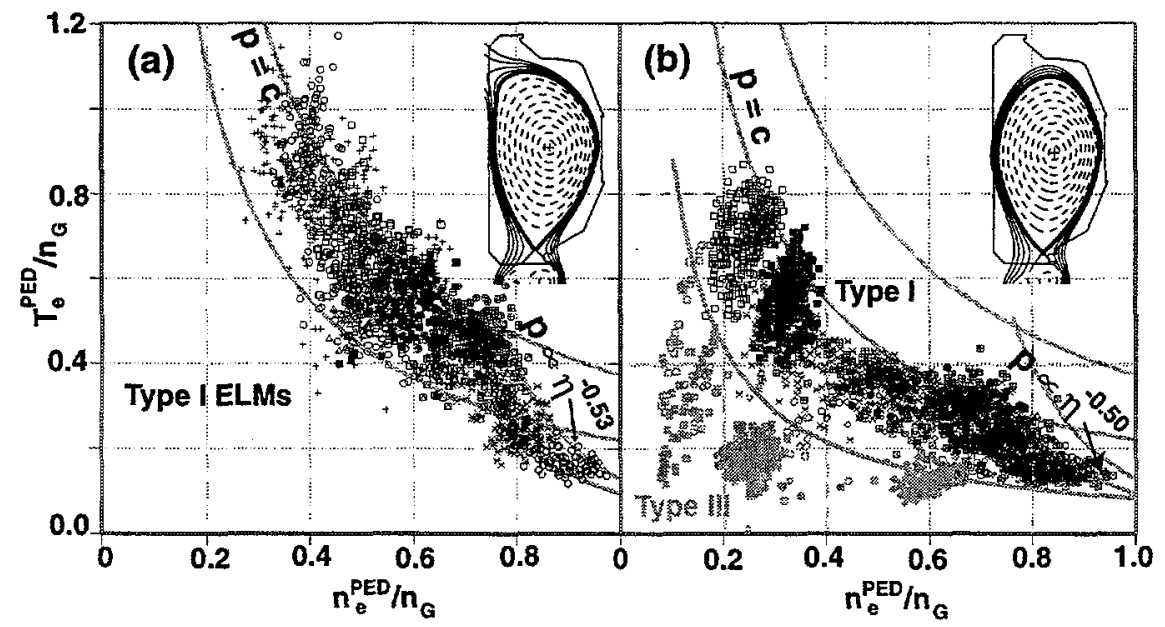

Fig. 4. Pedestal pressure begins to decrease at similar pedestal density normalized to the Greenwald density for (a) high and (b) low triangularity cases. Pressure decrease follows roughly $\eta^{-1 / 2}$ scaling such that near $n_{\mathrm{e}}^{\mathrm{PED}} / \mathrm{n}_{\mathrm{G}}=1$ the pressure is similar at high and low $\delta$. Type III ELMs, (b), are separated from Type I regime by a gap in pressure.
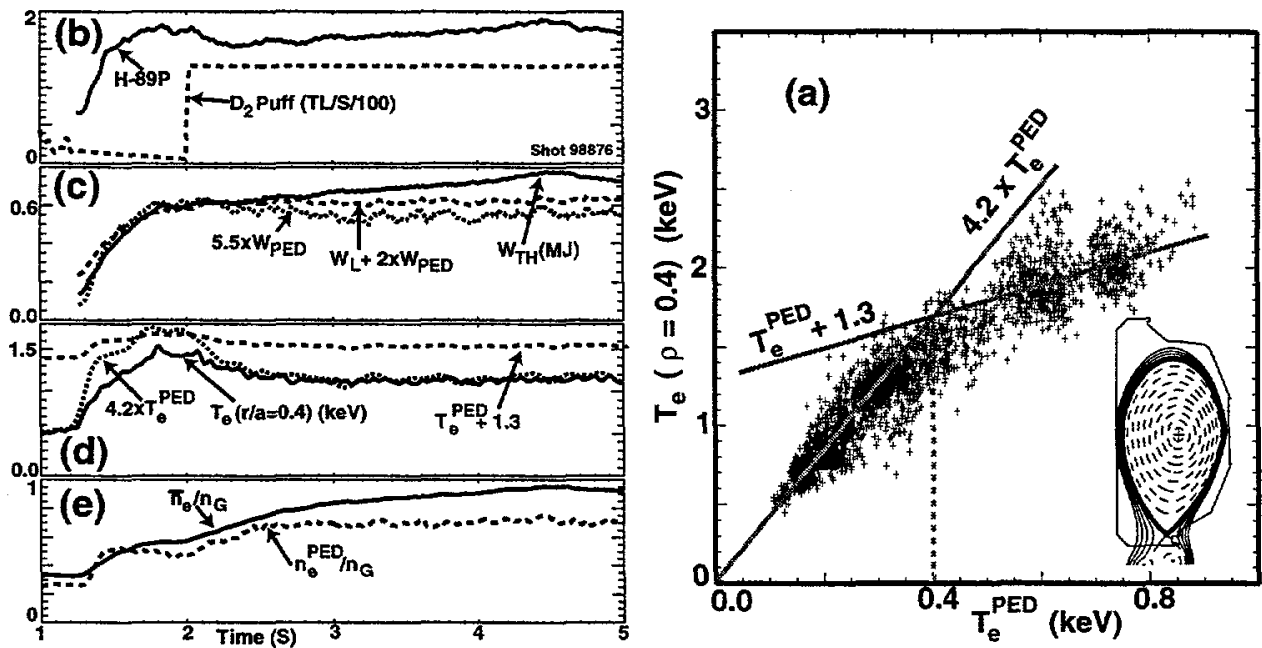

Fig. 5. Effect of pedestal parameters on core performance. (a) Temperature profiles are stiff at low temperature but additive at high temperature, (b) $\mathrm{H}$ factor recovers in high density gas puff fueled discharge with spontaneous density peaking, (c) rise in stored energy is not explained by either additive or proportional pedestal effect due to density profile change, (d) temperature profile is in the stiff regime after start of gas puffing, (e) density profile peaks, line average density reaches the Greenwald density. 